\title{
Binary-reaction spectroscopy of ${ }^{99,100} \mathrm{Mo}$ : Intruder alignment systematics in $N=\mathbf{5 7}$ and $N=\mathbf{5 8}$ isotones
}

\author{
P. H. Regan, ${ }^{1,2, *}$ A. D. Yamamoto, ${ }^{1,2}$ F. R. Xu, ${ }^{3}$ C. Y. Wu, ${ }^{4}$ A. O. Macchiavelli, ${ }^{5}$ D. Cline,${ }^{4}$ J. F. Smith, ${ }^{7}$ S. J. Freeman, ${ }^{7}$ \\ J. J. Valiente-Dobón, ${ }^{1}$ K. Andgren,${ }^{1,6}$ R. S. Chakrawarthy, ${ }^{7}$ M. Cromaz, ${ }^{5}$ P. Fallon, ${ }^{5}$ W. Gelletly, ${ }^{1}$ A. Gorgen, ${ }^{5}$ \\ A. Hayes, ${ }^{4}$ H. Hua, ${ }^{4}$ S. D. Langdown, ${ }^{1,2}$ I-Y. Lee, ${ }^{5}$ C. J. Pearson, ${ }^{1}$ Zs. Podolyák, ${ }^{1}$ R. Teng, ${ }^{4}$ and C. Wheldon ${ }^{1,8}$ \\ ${ }^{1}$ Department of Physics, University of Surrey, Guildford GU2 7XH, United Kingdom \\ ${ }^{2}$ Wright Nuclear Structure Laboratory, Yale University, New Haven, Connecticut 06520-8124, USA \\ ${ }^{3}$ Department of Technical Physics, Peking University, Beijing 100871, China \\ ${ }^{4}$ Nuclear Structure Research Laboratory, Department of Physics, University of Rochester, Rochester, New York 14627, USA \\ ${ }^{5}$ Nuclear Science Division, Lawrence Berkeley National Laboratory, Berkeley, California 94720, USA \\ ${ }^{6}$ Royal Institute of Technology, Department of Physics, S-106 91 Stockholm, Sweden \\ ${ }^{7}$ Department of Physics and Astronomy, The University of Manchester, Manchester M13 9PL, United Kingdom \\ ${ }^{8}$ Kernphysik II, GSI, Max-Planck-Straße 1, D-64291 Darmstadt, Germany
}

(Received 22 July 2003; published 20 October 2003)

\begin{abstract}
The near-yrast states of ${ }^{99,100}$ Mo have been studied following their population via a binary reaction between a ${ }^{136} \mathrm{Xe}$ beam and a thin, self-supporting ${ }^{100} \mathrm{Mo}$ target. The yrast sequence in ${ }^{100} \mathrm{Mo}$ has been extended to a tentative spin/parity $\left(20^{+}\right)$, while the decoupled band built on the $I^{\pi}=\frac{11}{2}-$ isomeric state in ${ }^{99}$ Mo has been extended through the first alignment up to a tentative spin/parity of $\left(\frac{43}{2}-\right)$. The results are compared with self-consistent, cranked-mean-field calculations using a Woods-Saxon potential. The alignment systematics of the intruder $h_{11 / 2}$ bands in the $N=57$ isotones from Mo $(Z=42)$ to $\mathrm{Cd}(Z=48)$ and the yrast sequences in their $N=58$ even-even neighbors are discussed. An overall picture emerges, where the alignment properties evolve from being due to positive-parity neutrons in the ${ }_{48}^{105} \mathrm{Cd}$ to predominantly $\left(g_{9 / 2}\right)^{2}$ proton crossings closer to the $Z=40$ subshell. Qualitatively, this can be explained by an increase in the quadrupole deformation and a simultaneous lowering of the proton Fermi surface in the $g_{9 / 2}$ shell with decreasing proton number. These data provide excellent examples of rotational-alignment phenomena in weakly deformed nuclei.
\end{abstract}

DOI: 10.1103/PhysRevC.68.044313

PACS number(s): 21.10.Re, 23.20.Lv, 27.60.+j

The cranked shell model (CSM) [1] and associated cranked-Woods-Saxon-Strutinsky mean-field [2-4] methods have been successful in interpreting the high-spin behavior in a wide range of well-deformed $(\beta \geqslant 0.25)$ nuclei. In particular, the predictions of rotational alignments of high- $j$ orbitals due to the Coriolis interaction in rare-earth nuclei are generally well reproduced [1]. In contrast, the applicability of the CSM to less deformed regions of the Segré chart remains less certain. Perhaps paradoxically, the Coriolis effects responsible for the rotational alignment phenomena associated with well-deformed rare-earth nuclei are arguably largest in systems with small intrinsic deformations [5]. This arises since the Coriolis matrix element has a linear dependence on $\hbar^{2} / \mathcal{I}$, where $\mathcal{I}$ is the moment of inertia [6].

Rotational-like sequences in the $N=57$ and $N=58$ isotones between the subshell closure at $Z=40(\mathrm{Zr})$ and the magic number at $Z=50(\mathrm{Sn})$ present particularly good laboratories with which to probe such Coriolis-driven alignment effects in weakly deformed nuclei (e.g., Refs. [7-16]). The odd- $A$, $N=57$ isotones systematically exhibit weakly deformed, decoupled bands associated with the population of the low- $\Omega$ components of the unique-parity $h_{11 / 2}$ orbital $[8,9]$. By comparing the observed increase in aligned angular momentum as the structures of these decoupled negative-parity se-

\footnotetext{
*Corresponding author. Email address: P.Regan@surrey.ac.uk
}

quences evolve with spin, it is possible to gain insight into the nature of the particles that are responsible for the alignment. Since the predicted alignment gains and crossing frequencies are also shape dependent, the study of these highspin processes can provide insight into the nuclear shape prior to the alignment, albeit in a model-dependent way (e.g., Refs. [7,14]). Related to this question, there has been recent discussion in the literature with regard to the evolution from vibrational to rotational-like sequences in the $A \sim 100-110$ region. This change in structure is argued to be associated with a stabilization of the nuclear quadrupole shape following the population of low $\Omega$, neutron $h_{11 / 2}$ orbitals, which, in the Nilsson scheme have equatorial trajectories for prolate shapes $[15,17]$.

The Coriolis interaction is largest for particles with large $j$ values and large intrinsic alignments on the rotation axis (i.e., small $\Omega$ values) [5]. Therefore as the atomic number is reduced and the Fermi surface falls lower in the $g_{9 / 2}$ subshell, one might expect an increased influence on alignments from the lower- $\Omega$ Nilsson components of the $g_{9 / 2}$ protons. This can be explained by the position of the proton Fermi surface changing from the mid-to-high $\Omega$ orbitals of the $g_{9 / 2}$ subshell for ${ }_{48}^{105} \mathrm{Cd}_{57}$ to the lower- $\Omega$ components for ${ }_{42}^{99} \mathrm{Mo}_{57}$. By comparison with the CSM, the alignment properties in the decoupled, $h_{11 / 2}$ structures in the $N=57$ isotones ${ }_{46}^{103} \mathrm{Pd}$ and ${ }_{48}^{105} \mathrm{Cd}$ have also been shown to demonstrate a strong dependence on the quadrupole deformation [9]. Specifically the observation 
of an alignment associated with a (predominantly) $g_{7 / 2}$ neutron pair has been proposed to explain the first band crossing in this structure in ${ }_{48}^{105} \mathrm{Cd}$. This is consistent with the small quadrupole deformation $\left(\beta_{2} \leqslant 0.15\right)$ expected for this "nearmagic" isotone. In contrast, a more complicated scenario has been suggested for ${ }_{46}^{103} \mathrm{Pd}$, where the alignment has been attributed to the simultaneous alignment of both $g_{7 / 2}$ neutrons and $g_{9 / 2}$ protons [9]. This is associated with an increase in deformation for ${ }^{103} \mathrm{Pd}$ compared to ${ }^{105} \mathrm{Cd}$ and a simultaneous lowering of the proton Fermi surface in to more easily 'alignable' orbitals [9], lower in the subshell. Following this trend, our recent study of ${ }_{44}^{101} \mathrm{Ru}$ revealed a sequence where the increase in alignment appears to come almost exclusively from the $g_{9 / 2}$ protons, with no evidence for any effect due to the $g_{7 / 2}$ neutron orbital [8].

Motivated by these aspects, we report new data pertinent to the alignment processes in the negative-parity intruder sequence in the $N=57$ isotone, ${ }_{42}^{99} \mathrm{Mo}$, and its even-even neighbor, ${ }^{100} \mathrm{Mo}$. This enables a complete picture of the alignment systematics of the $h_{11 / 2}$ bands in the even- $Z, N=57$ isotones ${ }_{42}^{99} \mathrm{Mo},{ }_{44}^{101} \mathrm{Ru},{ }_{46}^{103} \mathrm{Pd}$, and ${ }_{48}^{105} \mathrm{Cd}$ to be made. When compared with cranked-Woods-Saxon calculations, the overall results highlight the increasingly important impact of the $g_{9 / 2}$ protons on the alignment properties of these structures with decreasing proton number. These systematics provide excellent examples of multiple facets of rotational alignment phenonema in weakly deformed nuclei.

The rather neutron-rich nature of ${ }^{99} \mathrm{Mo}$ and ${ }^{100} \mathrm{Mo}$ make high-spin studies of these nuclei experimentally challenging. This is due to the lack of suitable choices for stable beamtarget combinations with which these systems can be populated via fusion-evaporation reactions. The spins, parities, and main spherical-basis single-particle parentage of the low-lying states in ${ }^{99} \mathrm{Mo}$ (including that of the $I^{\pi}=\frac{11-}{2}, T_{1 / 2}$ $=760 \mathrm{~ns}$ isomer at $684 \mathrm{keV}$ ) are however well established from charged-particle pick-up and stripping reactions (see, e.g., Ref. [18]). Previous work on the $h_{11 / 2}$ structure of ${ }^{99} \mathrm{Mo}$ has been carried out using backed-target deep-inelastic reactions [7]. It has also been studied via the weakly populated, $\alpha 2 n$ side-channel from our previous work on ${ }^{101} \mathrm{Ru}[8,17]$. The yrast sequence in ${ }^{100}$ Mo is known only to a tentative spin/parity of $12^{+}$, via both binary reaction [7] and light-ion induced fusion-evaporation [19] studies.

In the current work, the nuclei of interest were populated using heavy-ion binary reactions between a self-supporting, $\left(420 \mu \mathrm{g} / \mathrm{cm}^{2}\right){ }^{100}$ Mo target and a ${ }^{136} \mathrm{Xe}$ beam at an energy of $700 \mathrm{MeV}$. The beam was provided by the $88^{\prime \prime}$ cyclotron at the Lawrence Berkeley National Laboratory and had a natural pulsing of $\approx 2-3 \mathrm{~ns}$ width, separated by $64 \mathrm{~ns}$. Typical beam currents were the order of 1-2 pnA. Reaction $\gamma$ rays were detected using the GAMMASPHERE array [20], which, in this experiment, consisted of 102 Comptonsuppressed hyperpure germanium detectors. The binary fragments were detected using the position-sensitive gas-filled detector, CHICO [21], which enabled an event-by-event Doppler correction to be applied to the raw $\gamma$-ray data. The detection of co-planar events in CHICO allowed the separation of both beamlike and targetlike fragments by the measurement of their position relative to the beam direction. The

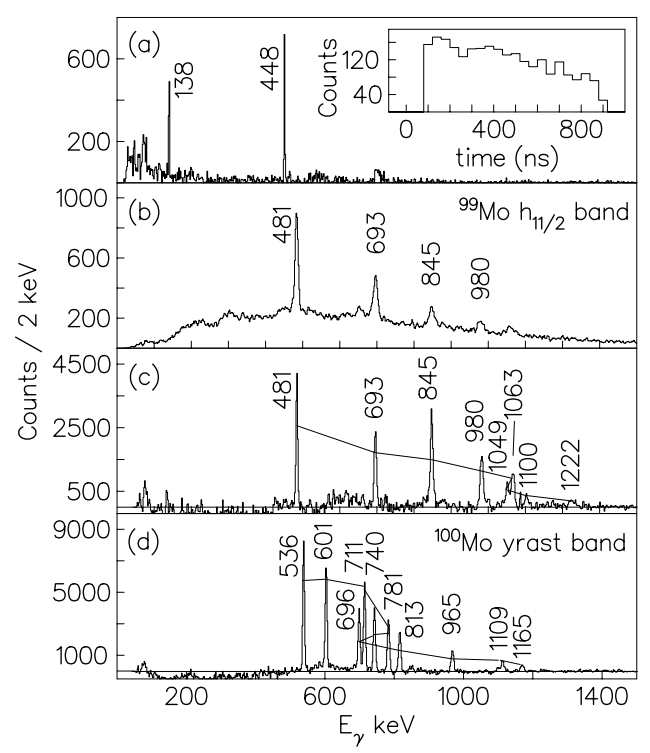

FIG. 1. (a) Delayed $\gamma$-ray spectrum in coincidence with the prompt $481 \mathrm{keV}, \frac{15}{2}^{-} \rightarrow \frac{11}{2}^{-}$transition in ${ }^{99} \mathrm{Mo}$. (b) Prompt $\gamma$-ray spectrum Doppler corrected for targetlike recoils and gated on delayed coincidences with the $448 \mathrm{keV} \frac{11}{2}^{-} \rightarrow \frac{7}{2}^{+}$transition depopulating the $E_{x}=684 \mathrm{keV}$ isomer in ${ }^{99} \mathrm{Mo}$. (c) Sum of double $\gamma$-ray gates showing the members of the $h_{11 / 2}$ band in ${ }^{99} \mathrm{Mo}$; (d) sum of double $\gamma$-ray gates showing the yrast band in ${ }^{100} \mathrm{Mo}$. The inset in (a) shows the time difference spectrum between the $448 \mathrm{keV}$ delayed transition and the 481,693 , and $845 \mathrm{keV}$ prompt $\gamma$ rays from the cascade which feed the $T_{1 / 2}=760 \mathrm{~ns}, I^{\pi}=\frac{11}{2}-$ isomer in ${ }^{99} \mathrm{Mo}$.

velocity of the targetlike fragments was angle dependent and calculated assuming two-body kinematics to vary between $3 \%$ and $11 \%$ of the speed of light.

The acquisition master trigger required that at least three prompt, Compton-suppressed $\gamma$ rays were detected in GAMMASPHERE within $\approx 50 \mathrm{~ns}$ of each other, together with two, co-planar binary fragments in CHICO. The heavy metal collimators were removed from the GAMMASPHERE Compton-suppression shields, thereby allowing a measure of the $\gamma$-ray fold for each event to be made. The data were sorted into standard $\gamma$-ray coincidence matrices and cubes which were subsequently analyzed using the RADWARE package [22]. A total of $900 \times 10^{6}$ suppressed germanium triples and higher-fold events were detected in coincidence with two co-planar binary fragments during the course of a four day experiment.

Using the temporal separation between prompt Dopplershifted transitions which feed isomeric states and those transitions which depopulate such isomers and decay from fragments stopped in the CHICO detector, time-correlated spectroscopy could be performed. In this way delayed gates were set on transitions depopulating the $T_{1 / 2}=760 \mathrm{~ns}, I^{\pi}$ $=\frac{11}{2}-$ isomeric state in ${ }^{99} \mathrm{Mo}$ [23] and the prompt transitions above the isomer could be clearly identified. Examples of spectra generated from this analysis are shown in Fig. 1, together with a sum of prompt, double-gated $\gamma$-ray coincidences on the $h_{11 / 2}$ band members in ${ }^{99} \mathrm{Mo}$ and the yrast band in ${ }^{100} \mathrm{Mo}$. The transitions were ordered in terms of their observed coincidence intensity relative to the lowest band 
TABLE I. $\gamma$-ray transitions (uncertainty $\pm 1 \mathrm{keV}$ ) identified in ${ }^{99} \mathrm{Mo}$ and ${ }^{100} \mathrm{Mo}$. The relative $\gamma$-ray intensities were taken from a $2 \mathrm{D}$ fit to the $\gamma-\gamma$ coincidence matrix using the program ESCL8R [22].

\begin{tabular}{cccccccc}
\hline \hline \multicolumn{3}{c}{${ }^{99} \mathrm{Mo}$} & & \multicolumn{5}{c}{${ }^{100} \mathrm{Mo}$} \\
$E_{\gamma}$ & $E_{i}, E_{f}$ & $\Gamma_{i}, I_{f}$ & $I_{\gamma}$ & $E_{\gamma}$ & $E_{i}, E_{f}$ & $I_{i}^{+}, I_{f}^{+}$ & $I_{\gamma}$ \\
\hline 481 & 1165,684 & $\frac{15}{2}, \frac{11}{2}$ & & 536 & 536,0 & 2,0 & \\
693 & 1858,1165 & $\frac{19}{2}, \frac{15}{2}$ & $19(1)$ & 601 & 1137,536 & 4,2 & $48(3)$ \\
845 & 2703,1858 & $\frac{23}{2}, \frac{19}{2}$ & $14(1)$ & 711 & 1848,1137 & 6,4 & $36(3)$ \\
980 & 3683,2703 & $\left(\frac{27}{2}, \frac{23}{2}\right)$ & $9(1)$ & 781 & 2629,1848 & 8,6 & $24(2)$ \\
1063 & 4746,3683 & $\left(\frac{31}{2}, \frac{27}{2}\right)$ & $4(1)$ & 740 & 3369,2629 & $(10,8)$ & $16(1)$ \\
1049 & 5795,4746 & $\left(\frac{35}{2}, \frac{31}{2}\right)$ & $2(1)$ & 696 & 4065,3369 & $(12,10)$ & $12(1)$ \\
1100 & 6895,5795 & $\left(\frac{39}{2}, \frac{35}{2}\right)$ & $2(1)$ & 813 & 4878,4065 & $(14,12)$ & $8(1)$ \\
1222 & 8117,6895 & $\left(\frac{43}{2}, \frac{39}{2}\right)$ & $1(1)$ & 965 & 5843,4878 & $(16,14)$ & $5(1)$ \\
& & & & 1109 & 6952,5843 & $(18,16)$ & $2(1)$ \\
& & & & 1165 & 8117,6952 & $(20,18)$ & $1(1)$ \\
\hline \hline
\end{tabular}

member (see Table I). The spin and parity assignments were made assuming that the observed transitions all have stretched E2 character. The energies of the transitions and their mutually coincident 'in-band' nature are consistent with similar $E 2$ cascades built on the yrast and decoupled bands in the neighboring isotones [8,9].

Figure 2 shows the azimuthal angle versus the time-offlight difference observed by CHICO. The beamlike and targetlike fragments are separated by the reaction kinematics and the yields for both are peaked around the expected laboratory grazing angles of $\sim 26^{\circ}$ and $48^{\circ}$, respectively. Figure 3 shows fold distributions for ${ }^{100} \mathrm{Mo}$ and nearby nuclei. Up to spin $8 \hbar$ in ${ }^{100}$ Mo the spectra are dominated by a low-fold reaction mechanism (assumed to be Coulomb excitation), while states above the $10^{+}$level (associated with the aligned $\left(\nu h_{11 / 2}\right)^{2}$ configuration [7]) have distributions shifted to significantly higher folds, associated with deep-inelastic collisions. The quasielastic two-neutron transfer to ${ }^{102} \mathrm{Mo}$ has a

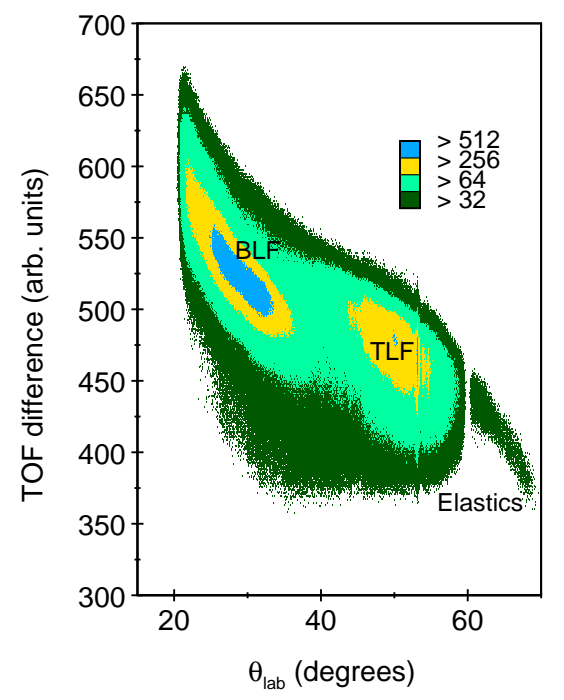

FIG. 2. (Color online) CHICO particle identification plot for the current work.

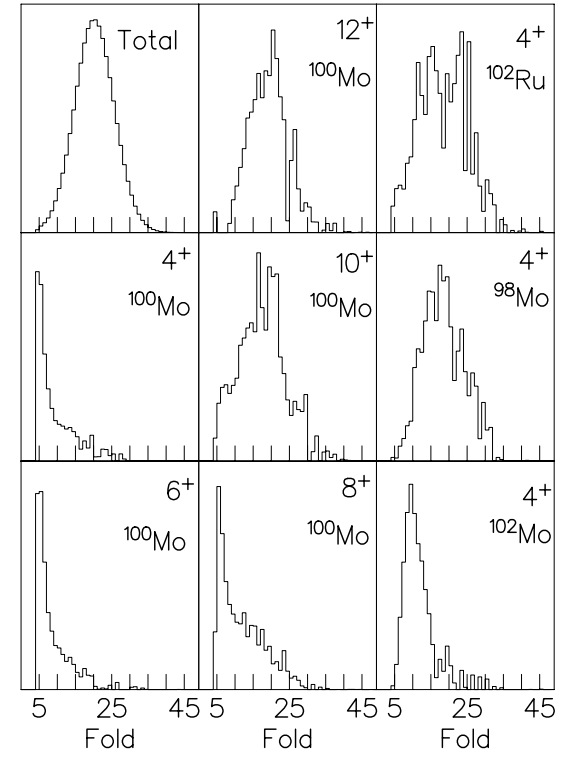

FIG. 3. Fold distributions gated on summed pairs of discrete double- $\gamma$-ray gates below the state of interest.

rather narrow fold distribution intermediate between that of Coulomb excitation and deep inelastic.

In our previous work using the ${ }^{96} \mathrm{Zr}\left({ }^{9} \mathrm{Be}, \alpha 2 n\right){ }^{99}$ Mo reaction [8], two new transitions at 979 and $1055 \mathrm{keV}$ were added to the $h_{11 / 2}$ decoupled sequence. The spectra in Fig. 1 confirm the 979-keV transition but also demonstrate that the reported $1055 \mathrm{keV}$ transition is in fact a merging of two separate in-band transitions, namely, those at $E_{\gamma}=1049$ and $1063 \mathrm{keV}$. This deconvolution is important as the backbending associated with the new ordering of these two transitions has a considerable impact on the deduced alignment.

A comparison of the rotational-like behavior in the decoupled $h_{11 / 2}$ bands in the $N=57$ isotones and their $N=58$ eveneven neighbors can be made in terms of the cranking model by extracting quantities such as the total aligned angular momentum $I_{x}$ and the quasiparticle angular momentum, $i_{x}[1]$. Figure 3 shows the comparison between the extracted $i_{x}$ for the yrast-sequences in the even-even cores of the $N=58$ isotones between $\mathrm{Mo}(Z=42)$ and $\mathrm{Cd}(Z=48)$, together with the same quantity for the decoupled $h_{11 / 2}$ sequence in the $N$ $=57$ isotonic neighbors. Note in each case the clear blocking effect associated with the population of the $h_{11 / 2}$ neutron orbital. The general trend is a 'mirroring' of the second alignment observed in the $N=58$ even-even core with the first alignment observed in the $N=57$ intruder band.

In order to compare the experimental data with state-ofthe-art, quantitive, microscopic theoretical predictions in the bandcrossing region, cranked-Woods-Saxon-Strutinsky calculations have been performed by means of total-Routhiansurface (TRS) calculations [3] in a three-dimensional deformation space $\left(\beta_{2}, \beta_{4}, \gamma\right)$ using the same procedure as outlined in Ref. [8]. At a given frequency, the deformation of a state is determined by minimizing the calculated TRS. Figure 4 shows the comparisons between the experimentally extracted total aligned angular momentum $I_{x}$ with those extracted from the angular momentum projections in the TRS calculations 


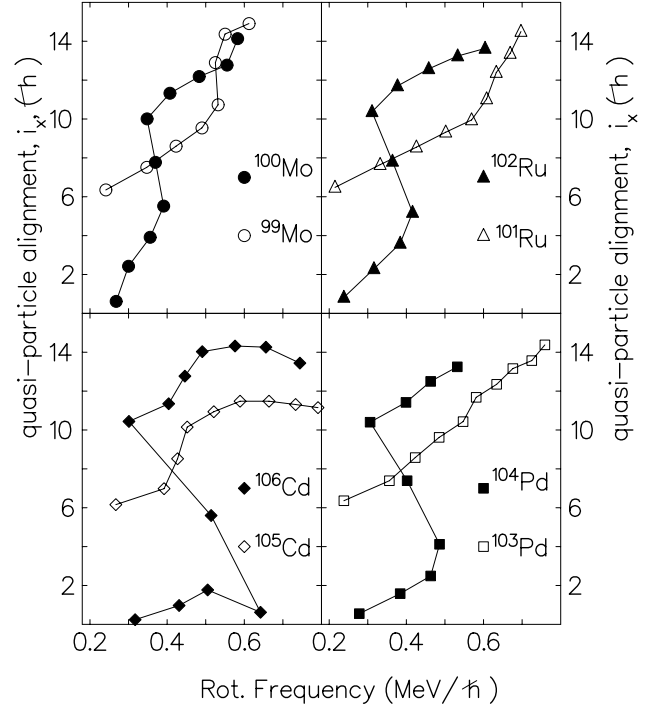

FIG. 4. Comparison of deduced quasiparticle alignments for the yrast bands in the even- $Z, N=58$ isotones between $\mathrm{Mo}$ and $\mathrm{Cd}$ and the decoupled $h_{11 / 2}$ structures in their $N=57$ neighbors. Harris parameters of $\mathcal{I}^{(0)}=7.0 \hbar^{2} / \mathrm{MeV}$ and $\mathcal{I}^{(1)}=15.0 \hbar^{4} / \mathrm{MeV}^{3}$ have been assumed in all cases $[8,9,11]$. The data are taken from the current work and Refs. [8,9,11,15,24].

for the $h_{11 / 2}$ bands in the $N=57$ isotones, ${ }_{42}^{99} \mathrm{Mo},{ }_{44}^{101} \mathrm{Ru}[8]$, ${ }_{46}^{103} \mathrm{Pd}[9,10]$, and ${ }_{48}^{105} \mathrm{Cd}[9,13]$.

The $h_{11 / 2}$ band in ${ }^{103} \mathrm{Pd}$ shows a gradual increase in alignment between $\omega \sim 0.2 \rightarrow 0.7 \mathrm{MeV} / \hbar$, which has been described in terms of a dual alignment of both $g_{7 / 2}$ neutrons and $g_{9 / 2}$ protons [9]. The comparatively lower rotational frequency of the (single component) first alignment in ${ }^{105} \mathrm{Cd}$ has been discussed in terms of a $\left(g_{7 / 2}\right)^{2}$ neutron crossing from blocking arguments and on the basis of the mirroring of the increase in $i_{x}$ in the yrast sequence in ${ }^{106} \mathrm{Cd}$ above the $\left(h_{11 / 2}\right)^{2}$ neutron alignment [11]. This implies that only one type of crossing [i.e., either $\left(g_{7 / 2}\right)^{2}$ neutrons or $\left(g_{9 / 2}\right)^{2}$ protons] is observed in the ${ }^{105} \mathrm{Cd}$ case. This alignment pattern is not as well reproduced as the lighter $N=57$ isotones in the calculations presented in Fig. 3. The $h_{11 / 2}$ band in ${ }^{103} \mathrm{Pd}$ appears to show a double crossing (as deduced from the large and continual increase in quasiparticle alignment over a wide frequency range). As pointed out in Ref. [9], this observation favors the argument that the alignment observed for ${ }^{105} \mathrm{Cd}$ is predominantly due to the $\left(g_{7 / 2}\right)^{2}$ neutrons [9]. This is consistent with the expectation that the $\left(\pi g_{9 / 2}\right)^{2}$ aligned configuration becomes more favored with decreasing proton number, while the crossing frequency of the $\left(g_{7 / 2}\right)^{2}$ neutrons is predicted to remain approximately constant (assuming similar deformations) for the $N=57$ isotones ${ }^{105} \mathrm{Cd}$ and ${ }^{103} \mathrm{Pd}$ (Fig. 5).

The experimentally deduced alignment gain for ${ }^{101} \mathrm{Ru}$ is reproduced by the theoretical calculations rather well. The combination of increased deformation compared to the heavier isotones, $\left({ }^{105} \mathrm{Cd}\right.$ and $\left.{ }^{103} \mathrm{Pd}\right)$, coupled to the lowering of the proton Fermi surface is demonstrated in the calculations to result in a predicted alignment which is dominated by the $\nu h_{11 / 2} \otimes\left(\pi g_{9 / 2}\right)^{2}$ configuration above the crossing.

The new data on ${ }^{99}$ Mo show an even more dramatic increase in alignment than that observed for ${ }^{101} \mathrm{Ru}$, with a

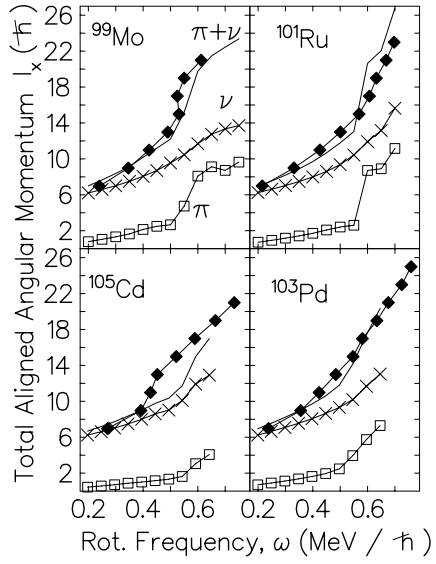

FIG. 5. Comparison of the experimentally extracted total aligned angular momentum $I_{x}$ for the $h_{11 / 2}$ bands in ${ }^{99} \mathrm{Mo},{ }^{101} \mathrm{Ru}$, ${ }^{103} \mathrm{Pd}$, and ${ }^{105} \mathrm{Cd}$ with the results of the TRS calculations. The open squares correspond to proton contributions, with the crosses representing the predicted neutron contribution. The solid lines are the total $I_{x}$ predicted by the TRS calculations and the large black diamonds are the values extracted from the experimental data.

backbend observed (rather than an upbend as seen in the heavier isotones). This is consistent with the weaker interaction expected for the $\left(\pi g_{9 / 2}\right)^{2}$ crossing associated with the lower- $\Omega$ orbitals, which reside closer to the Fermi surface for ${ }^{99} \mathrm{Mo}$. As with the $\nu h_{11 / 2}$ structure in ${ }^{101} \mathrm{Ru}$, the increased core deformation with respect to ${ }^{105} \mathrm{Cd}$ and ${ }^{103} \mathrm{Pd}$ is responsible for pushing the predicted $\left(\nu g_{7 / 2}\right)^{2}$ crossing up to higher frequencies, which probably explains why they are not observed in the current work. It is also worthy of note that the

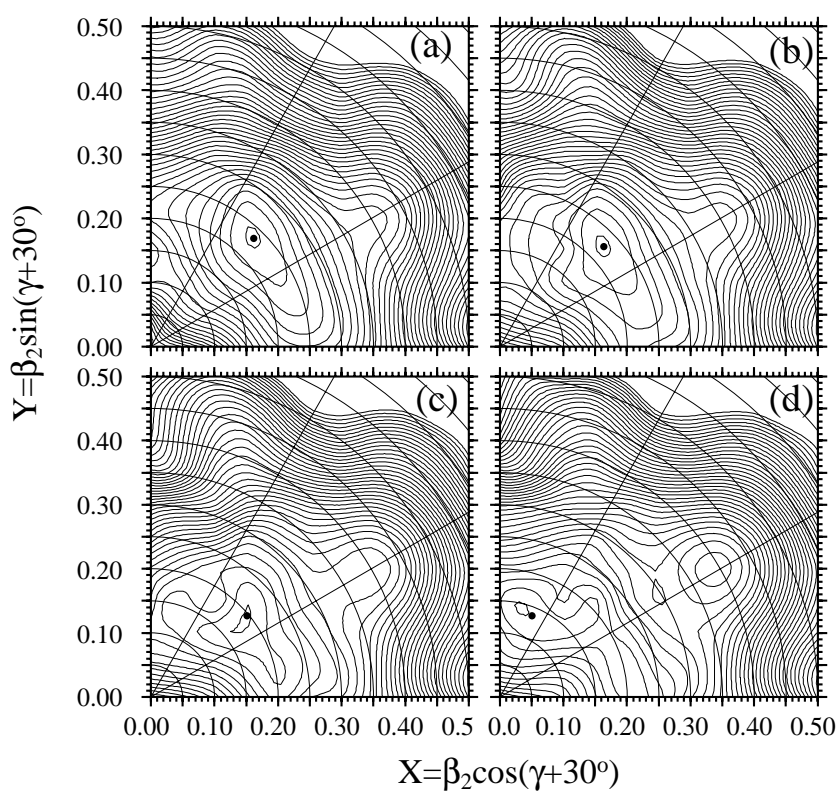

FIG. 6. Total Routhian Surface calculations for the lowest-lying negative-parity sequence in ${ }^{99} \mathrm{Mo}$. (a) $\omega=0.401 \mathrm{MeV} / \hbar\left(\beta_{2}=0.234\right.$, $\left.\beta_{4}=0.019, \gamma=+16.3^{\circ}\right)$; (b) $\omega=0.502 \mathrm{MeV} / \hbar\left(\beta_{2}=0.226, \beta_{4}=0.019\right.$, $\left.\gamma=+13.5^{\circ}\right) ; \quad$ (c) $\quad \omega=0.602 \mathrm{MeV} / \hbar \quad\left(\beta_{2}=0.197, \quad \beta_{4}=0.016\right.$, $\left.\gamma=+10.0^{\circ}\right)$; (d) $\omega=0.702 \mathrm{MeV} / \hbar\left(\beta_{2}=0.137, \beta_{4}=-0.006, \gamma=+38^{\circ}\right)$. The energy contour on these calculations is $200 \mathrm{keV}$. 
highest spin transition tentatively deduced for the yrast structure of ${ }^{100}$ Mo suggests the beginnings of an alignment at a similar frequency to that observed in the intruder band in the neighboring ${ }^{99}$ Mo.

The total Routhian surface calculations for the lowestlying negative-parity sequence in ${ }^{99}$ Mo are shown in Fig. 6. The predicted quadrupole deformation for this minimum has $\beta_{2} \sim 0.2$, but the minimum is soft with respect to both quadruple and triaxial degrees of freedom. The predicted effect of the $\left(g_{9 / 2}\right)^{2}$ proton alignment is to make this minimum even more $\gamma$ and $\beta$ soft, resulting in a rather, astable, lowdeformation configuration. It is perhaps rather surprising that the rotational-based CSM reproduces the experimental data so well in nuclei, which appear to have quasivibrational structure. We also note that at higher rotational frequencies $(\omega \approx 0.7 \mathrm{MeV} / \hbar$, corresponding to aligned spin values of $\approx 25 \rightarrow 30 \hbar$ ), a well-defined superdeformed minimum appears in the potential energy surface for ${ }^{99} \mathrm{Mo}$ at an axially symmetric deformation of $\beta_{2} \approx 0.4$, at an excitation energy of approximately $1 \mathrm{MeV}$ above the global minimum. A similar prediction has been made for the even-even neighbor ${ }^{100} \mathrm{Mo}$ [25]. The current experiment used an energy of approximately $25 \%$ above the nominal Coulomb barrier between the target and projectile [26], which using the semiclassical "rolling-mode" approximation [27] corresponds to a maximum expected value of the targetlike fragment intrinsic spin of $\sim 25 \hbar$. We note that this is close to what is observed discretely in the current experiment. For a heavier beam (such as ${ }^{208} \mathrm{~Pb}$ ) at a similar energy above the Coulomb barrier $(25-30 \%)$, the predicted rolling mode input spin is greater than $30 \hbar$, which may be enough to populate the predicted superdeformed minimum in both ${ }^{99} \mathrm{Mo}$ and ${ }^{100} \mathrm{Mo}$.

In summary, the near-yrast states of the $N=57$ and 58 nuclei ${ }^{99,100}$ Mo have been investigated using thin-target, heavy-ion-induced binary reactions. The partial decay schemes for these systems have been extended into the first band crossing region for the $h_{11 / 2}$ structure in ${ }^{99} \mathrm{Mo}$ and to the start of the second alignment in the yrast sequence in ${ }^{100} \mathrm{Mo}$. CSM calculations suggest both of these crossings are due to the favored rotational alignment of low- $\Omega$ components on the proton $g_{9 / 2}$ shell. When compared with the alignment systematics of the analogous structures in the neighboring $N=57$ and 58 isotones, and with cranked-Woods-SaxonStrutinksy calculations, a consistent picture emerges of the increasing importance of the low $\Omega g_{9 / 2}$ protons in the aligned configuration with decreasing proton number and the reduced effect of the $g_{7 / 2}$ neutrons with increasing core deformation.

\section{ACKNOWLEDGMENTS}

This work was supported by EPSRC (UK) and the U.S. Department of Energy, under Grant Nos. DE-FG02-91ER40609 and DE-AC03-76SF00098 and by the National Science Foundation. P.H.R. acknowledges support from Yale University via both the Flint and the Science Development Funds. A.D.Y., J.J.V.D., S.D.L., and Z.P. acknowledge the financial support of EPSRC. F.R.X. acknowledges support from the Major State Basic Research Development Program of China (Grant No. G2000077400) and the Chinese Ministry of Education.
[1] R. Bengtsson, S. Frauendorf, and F.-R. May, At. Data Nucl. Data Tables 35, 15 (1986).

[2] W. Nazarewicz, J. Dudek, R. Bengtsson, T. Bengtsson, and I. Ragnarsson, Nucl. Phys. A435, 397 (1985).

[3] W. Nazarewicz, R. Wyss, and A. Johnson, Phys. Lett. B 225, 208 (1989).

[4] W. Nazarewicz, M. A. Riley, and J. D. Garrett, Nucl. Phys. A512, 61 (1990).

[5] F. S. Stephens, R. M. Diamond, J. R. Leigh, T. Kammuri, and K. Nakai, Phys. Rev. Lett. 29, 438 (1972).

[6] R. F. Casten, Nuclear Structure from a Simple Perspective (Oxford University Press, New York, 2000), p. 364.

[7] P. H. Regan et al., Phys. Rev. C 55, 2305 (1997).

[8] A. D. Yamamoto et al., Phys. Rev. C 66, 024302 (2002).

[9] P. H. Regan, G. D. Dracoulis, G. J. Lane, P. M. Walker, S. S. Anderssen, A. P. Byrne, P. M. Davidson, T. Kibédi, A. E. Stuchbery, and K. C. Yeung, J. Phys. G 19, L157 (1993).

[10] B. M. Nyakó et al., Phys. Rev. C 60, 024307 (1999).

[11] P. H. Regan et al., Nucl. Phys. A586, 351 (1995).

[12] K. R. Pohl, P. H. Regan, J. E. Bush, P. E. Raines, D. P. Balamuth, D. Ward, A. Galindo-Uribarri, V. P. Janzen, S. M. Mullins, and S. Pilotte, Phys. Rev. C 53, 2682 (1996).
[13] D. Jerrestam et al., Nucl. Phys. A593, 162 (1995).

[14] H. Hua et al., Phys. Lett. B 562, 201 (2003).

[15] P. H. Regan et al., Phys. Rev. Lett. 90, 152502 (2003).

[16] P. H. Regan et al., Phys. Rev. C 49, 1885 (1994).

[17] P. H. Regan et al. in The Frontiers of Nuclear Structure, edited by P. Fallon and R. Clark, AIP Conf. Proc. No. 656 (AIP, Melville, NY, 2003), p. 422.

[18] S. Hirowatarai et al., Nucl. Phys. A714, 3 (2003).

[19] D. Hook, J. L. Durell, W. Gelletly, J. Lukasiak and W. R. Phillips, J. Phys. G 12, 1277 (1987).

[20] I-Y. Lee, Nucl. Phys. A520, 641c (1990).

[21] M. W. Simon et al., Nucl. Instrum. Methods Phys. Res. A 452, 205 (2000).

[22] D. C. Radford, Nucl. Instrum. Methods Phys. Res. A 361, 297 (1995).

[23] J. Dubuc, G. Kajrys, P. Lariviére, S. Pilotte, W. Del Bianco, and S. Monaro, Phys. Rev. C 37, 954 (1988).

[24] A. O. Macchiavelli et al., Phys. Rev. C 38, 1088 (1988).

[25] J. Skalski, S. Mizutori, and W. Nazarewicz, Nucl. Phys. A617, 282 (1997).

[26] C. Wheldon (private communication).

[27] R. Bock et al., Nukleonika 22, 529 (1977). 